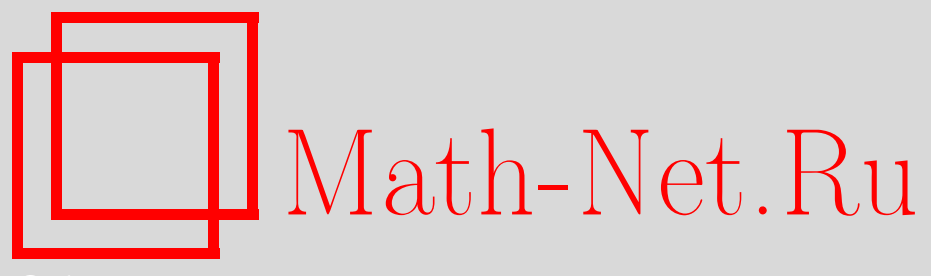

В. В. Штепин, Д. Л. Конашенков, Характеры и размерности представлений со старшим весом промежуточной группы Ли $D_{n-1 / 2}$, Изв. РАН. Сер. матем., 2014, том 78, выпуск 3, 205-224

DOI: https://doi.org/10.4213/im8034

Использование Общероссийского математического портала Math-Net.Ru подразумевает, что вы прочитали и согласны с пользовательским соглашением http://www . mathnet.ru/rus/agreement

Параметры загрузки:

IP: 107.22 .136 .117

26 апреля 2023 г., 13:22:55

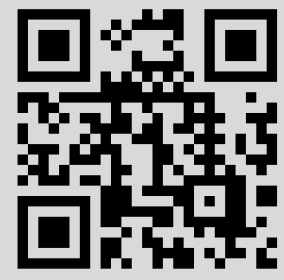


УДК 519.46

\author{
В. В. Штепин, Д. Л. Конашенков
}

\title{
Характеры и размерности представлений со старшим весом промежуточной группы Ли $D_{n-1 / 2}$
}

\begin{abstract}
Исследуются представления со старшим весом неполупростой комплексной группы Ли $D_{n-1 / 2}$, используемой для разделения кратных точек спектра в редукции $D_{n} \downarrow D_{n-1}$. В частности, найдены формулы для характеров и размерностей этих представлений, которые оказались аналогичными известным формулам Г. Вейля для классических групп Ли.

Библиография: 12 наименований.
\end{abstract}

Ключевые слова: полуклассические промежуточные группы Ли, конечномерные представления со старшим весом, правила ветвления, весовой базис, характер и размерность представления группы Ли.

\section{§ 1. Введение}

В настоящей работе продолжено изучение конечномерных представлений со старшим весом неполупростых промежуточных групп Ли (см. [1]-[3]). Они возникают естественным образом из следующей известной задачи спектрального анализа представлений классических групп Ли. Пусть $T_{m}$ - конечномерное неприводимое представление комплексной простой группы Ли $G_{n}$ одной из серий $A_{n}, B_{n}, C_{n}$ или $D_{n}, m$ - старший вес представления $T_{m}$. Требуется описать спектр сужения $T_{m}$ на максимальную одноименную подгруппу $G_{n-1}$ меньшего ранга $^{1}$ (т. е. $A_{n-1}, B_{n-1}, C_{n-1}$ или $D_{n-1}$ соответственно). Правило, описывающее разложение $\left.T_{m}\right|_{G_{n-1}}$ в прямую сумму неприводимых, называется $n p a в и$ лом ветвления. Для простых комплексных групп Ли правила ветвления были получены Г. Вейлем для унимодулярной серии (см. [4]), И. М. Гельфандом и М.Л. Цетлиным для двух ортогональных серий (см. [5]) и Д. П. Желобенко для симплектической серии (см. [6]). Отметим, что во всех четырех случаях спектр $\left.T_{m}\right|_{G_{n-1}}$ может содержать кратные точки.

Традиционный метод разделения кратных точек спектра (метод Гельфанда-Цетлина; см., например, [7, гл. 10]) предусматривает построение цепочки полупростых (или редуктивных) подгрупп

$$
G_{n-1}=G^{0} \subset G^{1} \subset G^{2} \subset \cdots \subset G^{s}=G_{n}
$$

такой, что спектр сужения каждого неприводимого $G^{i}$-подмодуля $T_{m}$ на $G^{i-1}$, $i=1,2, \ldots, s$, является простым. Однако этот метод является неприменимым

\footnotetext{
${ }^{1}$ Вложение $G_{n-1}$ в $G_{n}$ предполагается регулярным.
} 
к редукциям $A_{n} \downarrow A_{n-1}$ и $C_{n} \downarrow C_{n-1}$ (так как не существует подходящих в смысле правила ветвления промежуточных полупростых подгрупп между указанными парами групп Ли), в то время как в редукциях $B_{n} \downarrow B_{n-1}$ и $D_{n} \downarrow D_{n-1}$ этот метод приводит к построению базиса в пространстве представления $T_{m}$, не являющегося весовым. Более глубокая причина недостаточности использования цепочки вложений

$$
\mathrm{SO}(2, C) \subset \mathrm{SO}(3, C) \subset \cdots \subset \mathrm{SO}(2 n, C)
$$

указана во введении к работе [3].

В работах [1]-[3] было предложено обобщение метода Гельфанда-Цетлина, использующее неполупростые промежуточные подгруппы $G_{n-1 / 2}$ такие, что

$$
G_{n-1} \subset G_{n-1 / 2} \subset G_{n}
$$

Было показано, что “освобождения от кратностей" можно добиться не за счет разложения $T_{m}$ в прямую сумму подпредставлений $G_{n-1 / 2}$, а путем построения в $T_{m}$ фильтрации $\left(G_{n-1 / 2}\right)$-подпредставлений, разделяющей изоморфные компоненты $\left.T_{m}\right|_{G_{n-1}}$. Под фильтрацией $f$, разделяющей изоморфные компоненты $\left.T_{m}\right|_{G_{n-1}}$, мы подразумеваем фильтрацию $\left(G_{n-1 / 2}\right)$-подпредставлений в $T_{m}$ такую, что:

а) $f$ - фильтрация без кратностей (ее различные факторы попарно не изоморфны);

б) сужения факторов $f$ на $G_{n-1}$ имеют однократный спектр.

Построение искомой фильтрации $f$ можно вести как на уровне групп Ли, так и на уровне алгебр Ли, но в работах [1]-[3] применялся инфинитезимальный подход. Не углубляясь в детали, напомним основные этапы этого построения, так как это объясняет происхождение категории $\mathcal{L}$ представлений $G_{n-1 / 2}$ со старшим весом.

Пусть $V_{m}$ - конечномерный неприводимый $\mathfrak{g}_{n}$-модуль со старшим весом $m$, $\mathfrak{g}_{n}$ - алгебра Ли группы Ли $G_{n}$.

ОПРЕДЕЛЕНИЕ 1.1. Вектор $v \in V_{m}$, собственный относительно картановской подалгебры $\mathfrak{h}_{n}$ алгебры Ли $\mathfrak{g}_{n}$ и старший относительно $\mathfrak{g}_{n-1}$, называется полустариим.

Отметим, что наличие кратностей в сужении $\left.T_{m}\right|_{G_{n-1}}$ связано с наличием полустарших векторов одного и того же веса относительно $\mathfrak{h}_{n}$. Хотя алгебра Ли $\mathfrak{g}_{n-1 / 2}$ не полупроста, можно указать векторы в $V_{m}$, в определенном смысле старшие относительно $\mathfrak{g}_{n-1 / 2}$ (так называемые квазистаршие векторы). При подходящем выборе цепочки вложений $\mathfrak{g}_{n-1} \subset \mathfrak{g}_{n-1 / 2} \subset \mathfrak{g}_{n}$ (вложения $\mathfrak{d}_{n-1} \subset$ $\mathfrak{d}_{n-1 / 2} \subset \mathfrak{d}_{n}$ описаны в $\left.\S 3\right)$ определение квазистаршего вектора может быть дано единым образом для всех четырех серий.

ОПРЕДЕЛЕНИЕ 1.2 (ср. [8], [9]). Полустарший вектор $v \in V_{m}$, удовлетворяющий соотношениям $X_{i n} v=0, i=1,2, \ldots, n-1$, называется квазистаршим. 
Обозначим через $V_{m}^{+}$(соответственно, $V_{m}^{++}$) линейную оболочку множества полустарших (соответственно, квазистарших) векторов. Подпространство $V_{m}^{+}$будем называть пространством полустариих векторов (соответственно, $V_{m}^{++}-$пространством квазистариих векторов). В отличие от полустарших векторов квазистаршие векторы разделены на весовой диаграмме $V_{m}$, т. е. в $V_{m}^{++}$существует весовой базис $Q$, состоящий из квазистарших векторов с попарно различными весами относительно $\mathfrak{h}_{n}$ (см. [1, предложения $\left.2.4,2.5\right]$ и предложение 2.3 в [2] и [3]). Пусть на множестве $Q$ задана нумерация $\xi$ (линейный порядок) такая, что

$$
q_{1} \underset{\xi}{<} q_{2} \underset{\xi}{<} \cdots \underset{\xi}{<} q_{N}
$$

причем $q_{1}$ - квазистарший вектор старшего веса, $q_{N}$ - квазистарший вектор младшего веса среди всех квазистарших векторов. Пусть $f_{\xi}-$ фильтрация $\mathfrak{g}_{n-1 / 2}$-подмодулей $V_{m}$, в которой модуль $D_{i}$ порождается квазистаршими векторами $q_{1}, q_{2}, \ldots, q_{i}, i=1,2, \ldots, N$. Фильтрации $f_{\xi}$ поставим в соответствие присоединенный градуированный модуль

$$
D_{\xi}=\bigoplus_{i=1}^{N} D_{i} / D_{i-1} .
$$

В работах [1]-[3] для всех четырех картановских серий была доказана следующая

Tеорема 1.1. Существует нумерация $\xi$ множества $Q$ такая, ито соответствующая фильтрация $f_{\xi} \mathfrak{g}_{n-1 / 2}$-подмодулей $V_{m}$ разделяет изоморбные компоненты $\left.V_{m}\right|_{\mathfrak{g}_{n-1}}$.

Нумерация $\xi$, удовлетворяющая условиям теоремы 1.1, строится, вообще говоря, неоднозначно. Утверждение этой теоремы можно дополнить следующим предложением, которое показывает, что присоединенный градуированный модуль $D_{\xi}$ фактически не зависит от выбора $\xi$, т. е. имеет инвариантный смысл.

ПРЕДЛОЖЕНИЕ 1.1. Пусть $\xi$ и $\eta$ - нумерации множества $Q$ такие, что соответствующие фильтрации $f_{\xi} u f_{\eta}$ разделяют изоморфные компоненты $\left.V_{m}\right|_{\mathfrak{g}_{n-1}}$. Тогда $D_{\xi} \cong D_{\eta}$ (изоморфизм $\mathfrak{g}_{n-1 / 2}$-модулей $)$.

Доказательство предложения 1.1 для симплектической серии приведено в работе [9], для остальных серий доказательство аналогично. Следовательно, инвариантный смысл имеет также соответствующий $T_{m}$ присоединенный градуированный $G_{n-1 / 2}$-модуль $\bigoplus_{l \rightarrow m} T_{l}$, где представления $T_{l}$ действуют в факторпространствах фильтрации ${ }^{2} f$. Если учесть, что представление $T_{l}$ группы Ли $G_{n-1 / 2}$ является циклическим, порожденным единственным старшим вектором веса $l$, то понятно, что $T_{l}$ является представлением со старшим весом.

\footnotetext{
${ }^{2}$ По построению фильтрации $f$ каждый ее фактор порождается образом некоторого квазистаршего вектора, $l$ - старший вес этого вектора. Запись $l \rightarrow m$ означает, что вес $l$ подчинен $m$. Точный смысл условий подчинения см. в $\S 2$.
} 
Из определения фильтрации $f$ следует, что представления $T_{l_{1}}, T_{l_{2}}$, отвечающие различным весам $l_{1}, l_{2}$, подчиненным $m$, попарно не изоморфны.

Обозначим через $\mathcal{L}$ категорию представлений $T_{l}, l \rightarrow m$, групп Ли $G_{n-1 / 2}$, получаемых из всевозможных неприводимых представлений $T_{m}$ группы Ли $G_{n}$ как факторпредставления фильтраций, разделяющих изоморфные компоненты $\left.T_{m}\right|_{G_{n-1}}$. Мы называем $\mathcal{L}$ категорией представлений со старшим весом. Представления этой категории, как правило, приводимы, но неразложимы. Для неполупростых групп Ли $G_{n-1 / 2}$ эти представления имеют такое же значение, какое для полупростых групп Ли имеют конечномерные неприводимые представления. В связи с категорией $\mathcal{L}$ важное значение имеет решение следующих проблем:

1) описать множество старших весов представлений категории $\mathcal{L}$;

2) описать спектры сужений на $G_{n-1}$ представлений категории $\mathcal{L}$;

3) найти формулы для характеров и размерностей представлений категории $\mathcal{L}$ группы Ли $G_{n-1 / 2}$.

Для унимодулярной и симплектической серий все перечисленные проблемы были решены в работе [1]. В настоящей работе мы рассматриваем серию $D$ и соответствующую категорию представлений групп $D_{n-1 / 2}$ со старшим весом. Серия $B$ будет рассмотрена позже.

В $\S 2$ рассматриваются правила ветвления для редукции $G_{n} \downarrow G_{n-1}$, определяются промежуточные группы Ли $G_{n-1 / 2}$, описываются их разложения Леви-Мальцева. Основной здесь является теорема 2.1, гарантирующая возможность разделения изоморфных компонент представления $\left.T_{m}\right|_{G_{n-1}}$ посредством промежуточной подгруппы $G_{n-1 / 2}$. Мы показываем также, как цепочка редукций

$$
D_{1} \subset D_{2-1 / 2} \subset D_{2} \subset \cdots \subset D_{n-1} \subset D_{n-1 / 2} \subset D_{n}
$$

позволяет построить канонический весовой базис в пространстве $V_{m}$ представления $T_{m}$.

Группа Ли $D_{n-1 / 2}$ и ее алгебра Ли $\mathfrak{d}_{n-1 / 2}$ изучаются в $\S 3$, здесь описаны их свойства, важные с точки зрения теории представлений.

Следующий $\S 4$ посвящен доказательству формул для характеров представлений $\mathcal{L}(\lambda)$ группы Ли $D_{n-1 / 2}$.

Наконец, в 55 мы выводим формулу для размерностей вышеупомянутых конечномерных представлений категории $\mathcal{L}$. Отметим, что полученные нами формулы для характеров и размерностей представлений со старшим весом аналогичны известным формулам Г. Вейля для характеров и размерностей неприводимых представлений классических групп Ли [10].

\section{§ 2. Промежуточные группы Ли}

Мы будем рассматривать четыре серии комплексных простых групп Ли $A_{n}$, $B_{n}, C_{n}$ и $D_{n}$ и неполупростые группы Ли, занимающие промежуточное положение между классическими. Эти последние группы мы будем называть 
полуклассическими. Пусть $T_{m}$ - конечномерное неприводимое представление комплексной простой группы Ли $G_{n}$ одной из четырех упомянутых серий, $m-$ старший вес представления $T_{m}$. Напомним, что множество $P_{n}$ старших весов группы $G_{n}$ находится во взаимно однозначном соответствии с множеством классов эквивалентности ее конечномерных неприводимых представлений и в зависимости от серии имеет следующий вид:

$$
\begin{gathered}
P_{n}^{A}=P_{n}^{C}=\left\{\left(m_{1}, \ldots, m_{n}\right) ; m_{1} \geqslant m_{2} \geqslant \cdots \geqslant m_{n} \geqslant 0, m_{i} \in \mathbb{Z}\right\}, \\
P_{n}^{B}=\left\{\left(m_{1}, \ldots, m_{n}\right) ; m_{1} \geqslant m_{2} \geqslant \cdots \geqslant m_{n} \geqslant 0, m_{i} \in \mathbb{Z} \text { или } m_{i} \in \mathbb{Z}+\frac{1}{2}\right\}, \\
P_{n}^{D}=\left\{\left(m_{1}, \ldots, m_{n}\right) ; m_{1} \geqslant \cdots \geqslant m_{n-1} \geqslant\left|m_{n}\right|, m_{i} \in \mathbb{Z} \text { или } m_{i} \in \mathbb{Z}+\frac{1}{2}\right\} .
\end{gathered}
$$

Мы обозначим через $G_{n-1}$ соответствующие максимальные одноименные подгруппы меньшего ранга, т. е. $A_{n-1}, B_{n-1}, C_{n-1}$ и $D_{n-1}$ соответственно. Как было отмечено во введении, правила ветвления для серий Картана известны уже давно. Замечательно то, что для всех четырех серий их можно записать в общем виде

$$
\left.T_{m}\right|_{G_{n-1}}=\bigoplus \bigoplus_{l \rightarrow m}\left(\bigoplus_{t \rightarrow l} T_{t}\right), \quad m \in P_{n},
$$

где суммирование ведется по всем весам $l$, значения которых будут определены ниже, и $t \in P_{n-1}$. Стрелками обозначены определенные условия подчинения для старших весов. Эти условия различны для каждой из серий и могут быть описаны системами неравенств

$$
\begin{aligned}
& l \rightarrow m\left\{\begin{array}{l}
A: m_{1}-l_{1}=m_{2}-l_{2}=\cdots=m_{n}-l_{n} \geqslant 0, \\
C: m_{1} \geqslant l_{1} \geqslant m_{2} \geqslant l_{2} \geqslant \cdots \geqslant l_{n-1} \geqslant m_{n} \geqslant l_{n}, \\
B: m_{1} \geqslant l_{1} \geqslant m_{2} \geqslant l_{2} \geqslant \cdots \geqslant l_{n-1} \geqslant m_{n} \geqslant\left|l_{n}\right|, \\
D: m_{1} \geqslant l_{1} \geqslant m_{2} \geqslant l_{2} \geqslant \cdots \geqslant m_{n-1} \geqslant l_{n-1} \geqslant\left|m_{n}\right| ;
\end{array}\right. \\
& t \rightarrow l \Longleftrightarrow\left\{\begin{array}{l}
A, C: l_{1} \geqslant t_{1} \geqslant l_{2} \geqslant t_{2} \geqslant \cdots \geqslant l_{n-1} \geqslant t_{n-1} \geqslant l_{n}, \\
B: l_{1} \geqslant t_{1} \geqslant l_{2} \geqslant t_{2} \geqslant \cdots \geqslant l_{n-1} \geqslant t_{n-1} \geqslant\left|l_{n}\right|, \\
D: l_{1} \geqslant t_{1} \geqslant l_{2} \geqslant t_{2} \geqslant \cdots \geqslant l_{n-1} \geqslant\left|t_{n-1}\right| .
\end{array}\right.
\end{aligned}
$$

Правило ветвления, записанное в общем виде (2.1), позволяет выдвинуть гипотезу о том, что пространство $\bigoplus_{t \rightarrow l} T_{t}$ может быть наделено структурой не только $G_{n-1}$-модуля, но также структурой модуля над некоторой подгруппой Ли, промежуточной между $G_{n-1}$ и $G_{n}$. В терминологии И. М. Гельфанда и А. В. Зелевинского (см. [11]) это означает, что модуль $\bigoplus_{t \rightarrow l} T_{t}$ обладает "скрытой симметрией". Мы обозначаем эту группу $G_{n-1 / 2}$. Определение группы $G_{n-1 / 2}$ может быть дано более точно.

ОПРедЕЛЕНИЕ 2.1. Назовем стабилизатор ненулевого вектора (в случаях $A$ или $C$ ) и стабилизатор ненулевого изотропного вектора (в случаях $B$ или $D$ ) в пространстве стандартного представления группы Ли $G_{n}$ nолуклассической 
промежуточной группой Ли и обозначим $G_{n-1 / 2}$. Стандартным называют представление со старшим весом $(1,0, \ldots, 0) \in P_{n}$.

При таком определении условие $G_{n-1} \subset G_{n-1 / 2} \subset G_{n}$, очевидно, выполнено. Следующее предложение описывает внутреннее строение групп Ли $G_{n-1 / 2}$.

ПреДЛОжение 2.1. Группы Ли $G_{n-1 / 2}$ не редуктивны. Разложения ЛевиМальцева групп Ли $G_{n-1 / 2}$ серий $A, B$ и $C$ описываются следующим образом:

$$
\begin{gathered}
A_{n-1 / 2} \simeq A_{n-1} \ltimes \mathbb{C}^{n}, \quad B_{n-1 / 2} \simeq B_{n-1} \ltimes \mathbb{C}^{2 n-1}, \\
C_{n-1 / 2} \simeq C_{n-1} \ltimes \mathbb{H}_{2 n-1},
\end{gathered}
$$

где $\mathbb{H}_{2 n-1}$ - группа Гейзенберга размерности $2 n-1, \ltimes-$ полупрямое произведение групп Ли.

ДокаЗАТЕЛЬСТво см. в [1], [2].

Группа Ли $D_{n-1 / 2}$ подробно рассматривается в следующем параграфе.

Теорема 2.1. Пусть $T_{m}$ - конечномерное неприводимое представление группь Ли $G_{n}$ со стариим весом $m \in P_{n}$. Тогда сужение $T_{m}$ на $G_{n-1}$ допускает разделение изоморфных компонент посредством промежуточной nодгруппь $G_{n-1 / 2}$.

ДоКАЗАТЕЛЬСТВо см. в [1] для унимодулярной и симплектической серий, в [2] для серии $B$, в [3] для серии $D$.

Под разделением изоморфных компонент мы подразумеваем построение фильтрации $f\left(G_{n-1 / 2}\right)$-подпредставлений в $T_{m}$ такой, что:

a) $f$ - фильтрация, свободная от кратностей (т. е. ее различные факторы попарно не изоморфны);

б) сужения факторов фильтрации $f$ на $G_{n-1}$ имеют простой спектр.

Отметим, что если условия а) и б) выполняются, то изоморфные неприводимые компоненты представления $\left.T_{m}\right|_{G_{n-1}}$ можно различать, относя их к неизоморфным факторам фильтрации $f$.

Заметим, кроме того, что если промежуточные подгруппы в этом определении просты, то наше разделение изоморфных компонент сводится к традиционному методу Гельфанда-Цетлина. В этом случае можно заменить факторы фильтрации прямыми слагаемыми, а фильтрацию $f$ - прямой суммой. Однако, как это следует из предложения 2.1 , все группы $G_{n-1 / 2}$ не редуктивны и, следовательно, не полупросты.

Напомним, что условия подчинения (2.2), (2.3) для ортогональных серий, доказанные Гельфандом и Цетлиным для цепочки редукций

$$
D_{1} \subset B_{1} \subset D_{2} \subset \cdots \subset D_{n-1} \subset B_{n-1} \subset D_{n},
$$

становятся некорректными для цепочки редукций

$$
D_{1} \subset D_{2-1 / 2} \subset D_{2} \subset \cdots \subset D_{n-1} \subset D_{n-1 / 2} \subset D_{n}
$$

(см. [3, пример 2.1]). 
Можно ли "исправить" правило ветвления так, чтобы оно соответствовало последней цепочке редукций? Ответ был получен в работе [3] и имеет следующий вид:

$$
\begin{gathered}
l \rightarrow m \Longleftrightarrow m_{1} \geqslant l_{1} \geqslant m_{2} \geqslant \cdots \geqslant m_{n-1} \geqslant l_{n-1} \geqslant-m_{n}, \\
t \rightarrow l \Longleftrightarrow l_{1} \geqslant t_{1} \geqslant l_{2} \geqslant t_{2} \geqslant \cdots \geqslant l_{n-2} \geqslant t_{n-2} \geqslant \nu\left(m_{n}, l_{n-1}\right) \\
\geqslant l_{n-1} \geqslant t_{n-1} \geqslant l_{n-1}+m_{n}-\mu\left(m_{n-1}, t_{n-2}\right),
\end{gathered}
$$

где введены обозначения $\nu(a, b)=\max (a, b), \mu(a, b)=\min (a, b)$.

ОПРЕДЕЛЕНИЕ 2.2. Будем говорить, что тройка весов $t \in P_{n-1}^{D}, l \in P_{n-1}^{D}$, $m \in P_{n}^{D}$ образует кортеж $\{t, l, m\}$, если $t \rightarrow l \rightarrow m$, где условия подчинения весов такие же, как в (2.6).

В [3, следствие 3.1] был построен весовой базис $\{w(t, l, m),\{t, l, m\}-$ кортеж $\}$ пространства $V_{m}^{+}$, состоящий из полустарших векторов $w(t, l, m)$. Пусть $W(t, l, m)$ - циклический $\mathfrak{d}_{n-1}$-подмодуль $V_{m}$, порожденный вектором $w(t, l, m)$, $t \rightarrow l \rightarrow m$. Согласно [3, предложение $2.2,($ б)] подмодули $W(t, l, m)$ неприводимы и $V_{m}$ разлагается в их прямую сумму:

$$
\left.V_{m}\right|_{\mathfrak{o}_{n-1}}=\bigoplus_{t \rightarrow l \rightarrow m} W(t, l, m)
$$

Рассуждая индуктивно, мы можем считать, что в подпространствах $W(t, l, m)$ уже построены весовые базисы. Таким образом, мы получаем канонический весовой базис $\mathfrak{d}_{n}$-модуля $V_{m}$. Термин "канонический" означает, что этот базис связан с цепочкой однократных редукций (2.5). Цепочка Гельфанда-Цетлина (2.4) также содержит однократные редукции, но, как хорошо известно, приводит к построению базиса пространства $V_{m}$, не являющегося весовым.

\section{§ 3. Группа Ли $D_{n-1 / 2}$ и ее алгебра Ли}

Комплексной простой группой Ли типа $D_{n}$ называют собственную ортогональную группу $\mathrm{SO}(2 n)$, состоящую из всех ортогональных преобразований комплексного $2 n$-мерного пространства $\mathbb{C}^{2 n}$, не изменяющих ориентацию.

Зафиксируем в $\mathbb{C}^{2 n}$ базис $e_{1}, e_{2}, \ldots, e_{n}, e_{-n}, \ldots, e_{-2}, e_{-1}$ и скалярное произведение, определяемое формулой

$$
(x, y)=x_{1} y_{-1}+\cdots+x_{n} y_{-n}+x_{-n} y_{n}+\cdots+x_{-1} y_{1} .
$$

Тогда группа $\mathrm{SO}(2 n)$ состоит из всех элементов $g$, удовлетворяющих матричному уравнению

$$
\sigma^{-1} g \sigma=\left(g^{T}\right)^{-1},
$$

где $\sigma$ - матрица, все элементы побочной диагонали которой равны 1 , а все остальные элементы равны нулю. Промежуточную подгруппу $D_{n-1 / 2}$ можно определить как стабилизатор ненулевого изотропного вектора в пространстве 
стандартного представления группы Ли $\mathrm{SO}(2 n)$. Как замкнутая подгруппа группы Ли промежуточная подгруппа $D_{n-1 / 2}$ является комплексной группой Ли.

Зафиксируем выбор вложений $\mathrm{SO}(2 n-2) \subset D_{n-1 / 2} \subset \mathrm{SO}(2 n)$ следующим образом. Подгруппа $D_{n-1 / 2}$ группы Ли $\mathrm{SO}(2 n)$ является стабилизатором $n$-го базисного вектора $e_{n}$ пространства $\mathbb{C}^{2 n}$, а $\mathrm{SO}(2 n-2)$ наряду с $e_{n}$ оставляет неподвижным также базисный вектор $e_{-n}$. Аналогично определяются вложения алгебр Ли $\mathfrak{s o}(2 n-2) \subset \mathfrak{d}_{n-1 / 2} \subset \mathfrak{s o}(2 n)$. Более точно, алгебру Ли $\mathfrak{s o}(2 n)$ (типа $\left.\mathfrak{d}_{n}\right)$ группы Ли $\mathrm{SO}(2 n)$ мы будем отождествлять с алгеброй матриц $\left\|a_{i j}\right\| \in \mathfrak{g l}(2 n)$, удовлетворяющих соотношению

$$
a_{i j}=-a_{-j,-i}, \quad i, j \in\{-n, \ldots,-1,1,2, \ldots, n\} .
$$

Она порождена $n(2 n-1)$ образующими

$$
X_{i j}=E_{i j}-E_{-j,-i},
$$

где $E_{i j}$ - образующие $\mathfrak{g l}(2 n)$ (матричные единицы).

Коммутационные соотношения в $\mathfrak{s o}(2 n)$ являются следствием (2.4) и коммутационных соотношений в $\mathfrak{g l}(2 n)$ и имеют вид

$$
\left[X_{i j}, X_{k l}\right]=\delta_{k, j} X_{i l}-\delta_{i, l} X_{k j}+\delta_{-l, j} X_{k,-i}-\delta_{k,-i} X_{-j, l},
$$

где $\delta_{\alpha, \beta}-$ символ Кронекера.

Подалгебра $\mathfrak{d}_{n-1 / 2}$ выделяется из алгебры Ли $\mathfrak{s o}(2 n)$ наложением условий $a_{i n}=0, a_{-n, i}=0, i= \pm 1, \ldots, \pm n$, a $\mathfrak{s o}(2 n-2)$ выделяется из $\mathfrak{d}_{n-1 / 2}$ с помощью соотношений $a_{n j}=0, a_{j,-n}=0, j= \pm 1, \ldots, \pm n$.

Подалгебра Картана $\mathfrak{h}_{n}$ алгебры Ли $\mathfrak{s o}(2 n)$ порождена образующими $X_{i i}$, $1 \leqslant i \leqslant n$. Пусть $R_{n}$ - система корней алгебры Ли $\mathfrak{s o}(2 n), P\left(R_{n}\right)$ - ее группа весов. Стандартный базис $B$ в $P\left(R_{n}\right)$ образуют элементы $\varepsilon_{1}, \ldots, \varepsilon_{n} \in \mathfrak{h}_{n}^{*}$, определенные равенствами $\varepsilon_{i}\left(X_{j j}\right)=\delta_{i j}, 1 \leqslant i, j \leqslant n$. Тогда

$$
R_{n}=\left\{ \pm \varepsilon_{i} \pm \varepsilon_{j}, 1 \leqslant i<j \leqslant n\right\}
$$

и простыми корнями системы $R_{n}$ являются

$$
\alpha_{1}=\varepsilon_{1}-\varepsilon_{2}, \quad \ldots, \quad \alpha_{n-1}=\varepsilon_{n-1}-\varepsilon_{n}, \quad \alpha_{n}=\varepsilon_{n-1}+\varepsilon_{n} .
$$

Система корней $R_{n-1 / 2}$ алгебры Ли $\mathfrak{d}_{n-1 / 2}$ состоит из векторов:

$$
R_{n-1 / 2}=\left\{\alpha \in R_{n} \mid\left(\alpha, \varepsilon_{n}\right) \geqslant 0\right\},
$$

где $(\cdot, \cdot)$ - произвольное скалярное произведение в $\mathfrak{h}_{n}^{*}$, инвариантное относительно группы Вейля. Соответственно, система корней $R_{n-1}$ алгебры Ли $\mathfrak{s o}(2 n-2)$ состоит из следующих корней:

$$
R_{n-1}=\left\{\alpha \in R_{n} \mid\left(\alpha, \varepsilon_{n}\right)=0\right\} .
$$

Следующее предложение описывает свойства группы Ли $D_{n-1 / 2}$, важные с точки зрения теории представлений. 
ПреДЛОЖенИЕ 3.1. (i) Группа Ли $D_{n-1 / 2}$ является экспоненциалъной.

(ii) Группа Ли $D_{n-1 / 2}$ не редуктивна, ее разложение Леви-Малъцева описъвается формулой

$$
D_{n-1 / 2} \simeq \mathrm{SO}(2 n-2) \ltimes \mathbb{C}^{2 n-2},
$$

где $\mathbb{C}^{2 n-2}$ - аддитивная группа Ли, $\ltimes$ - полупрямое произведение групп Ли.

ДокАзАтЕльство. Сначала докажем утверждение (i). Всякая матрица $g$ из $D_{n-1 / 2}$ может быть приведена к жордановой нормальной форме с жордановыми клетками, отвечающими собственным значениям матрицы $g$. Пусть $K-$ жорданова клетка, отвечающая $\lambda \in \mathbb{C}^{*}$. Тогда, вынося скалярный множитель, получим

$$
K=\operatorname{diag}(\lambda, \lambda, \ldots, \lambda)(E+N)
$$

где $N$ - нильпотентная матрица, все ненулевые элементы которой расположены над главной диагональю и равны $\lambda^{-1}$. Пользуясь представлением для $\ln (E+N)$ в виде степенного ряда (который в данном случае конечен, поэтому сходится), мы найдем матрицу $Z$ такую, что $\ln (E+N)=Z$, откуда $E+N=\exp Z$. Скалярный множитель также представим в виде экспоненты:

$$
\operatorname{diag}(\lambda, \lambda, \ldots, \lambda)=\exp D
$$

где $D$ - также скалярная матрица. Тогда будем иметь

$$
K=\exp D \cdot \exp Z=\exp (D+Z)
$$

ввиду того, что $D$ и $Z$ коммутируют. Если теперь $g(t)$ - однопараметрическая подгруппа в $D_{n-1 / 2}$ и $g(t)=\exp (t X)$, то $X \in \mathfrak{d}_{n-1 / 2}$ по определению алгебры Ли. Следовательно, $\exp \left(\mathfrak{d}_{n-1 / 2}\right)=D_{n-1 / 2}$, что и требовалось доказать.

Доказательство утверждения (ii) приведено в [3, предложение 1.1].

СлЕДСтвиЕ 3.1. Алгебра Ли $\mathfrak{d}_{n-1 / 2}$ имеет нетривиалъный радикал $r$, изоморфный коммутативной алгебре Ли размерности $2 n-2$, при этом

$$
\mathfrak{d}_{n-1 / 2} \simeq \mathfrak{s o}(2 n-2) \dot{+} r,
$$

где $\dot{+}-$ полупрямая сумма алгебр Ли.

\section{§ 4. Характеры представлений $\mathcal{L}(\lambda)$ со старшими весами $\left(\lambda_{1}, \ldots, \lambda_{n-1}, \lambda_{n}, \lambda_{n}\right)$}

Пусть $V_{m}$ - конечномерный неприводимый $\mathfrak{d}_{n}$-модуль. Как уже известно, сужение $V_{m}$ на подалгебру $\mathfrak{d}_{n-1}$ вполне приводимо и может содержать кратные точки спектра. Желание разделить изоморфные компоненты $\left.V_{m}\right|_{\mathfrak{a}_{n-1}}$ привело 
нас к построению фильтрации $f$ конечномерных $\mathfrak{d}_{n-1 / 2}$-подмодулей $V_{m}$. Каждый фактор $f$ является циклическим модулем, порожденным образом единственного квазистаршего вектора ${ }^{3} q_{\lambda}$, где $\lambda \rightarrow m$ (см. $\left.\S 1,2\right)$. Если $m$ пробегает множество старших весов $P_{n}^{D}$, то множество $\left(\mathfrak{d}_{n-1 / 2}\right)$-факторов фильтраций, разделяющих изоморфные компоненты $\mathfrak{d}_{n-1}$-модулей $V_{m}$, образует категорию $\mathcal{L}$ представлений со старшим весом алгебры Ли $\mathfrak{d}_{n-1 / 2}$. Модули категории $\mathcal{L}$, вообще говоря, не являются неприводимыми, но всегда неразложимы. Вернемся к проблемам, анонсированным во введении.

Согласно [3, следствие 4.2] объекты категории $\mathcal{L}$, рассматриваемые с точностью до изоморфизма $\mathfrak{d}_{n-1 / 2}$-модулей, находятся во взаимно однозначном соответствии с множеством $\widetilde{P}_{n+1}-$ множеством целочисленных векторов с компонентами $\lambda_{1}, \lambda_{2}, \ldots, \lambda_{n-1}, m_{n-1}, m_{n}$, удовлетворяющими системе неравенств

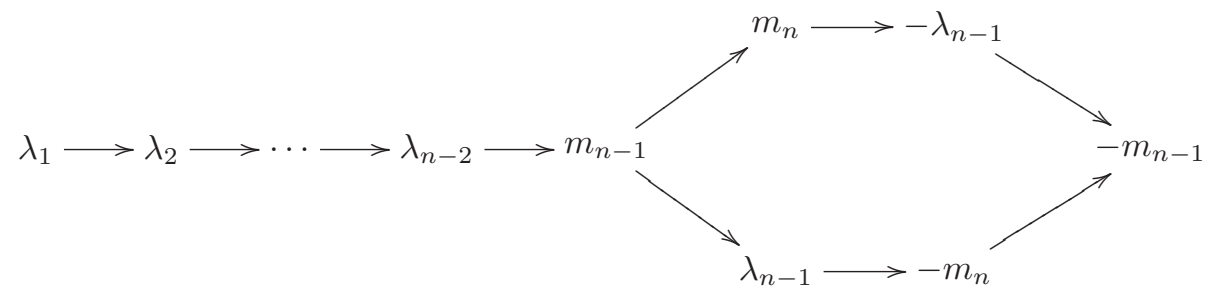

где стрелка означает знак $\geqslant$. Следовательно,

$$
\mathcal{L}=\left\{\mathcal{L}\left(\lambda_{1}, \lambda_{2}, \ldots, \lambda_{n-1}, m_{n-1}, m_{n}\right) ;\left(\lambda_{1}, \lambda_{2}, \ldots, \lambda_{n-1}, m_{n-1}, m_{n}\right) \in \widetilde{P}_{n-1}\right\} .
$$

Наличие параметров $m_{n-1}$ и $m_{n}$ среди компонент веса модуля $\mathcal{L}(\lambda)$ обусловлено тем фактом, что фильтрация, разделяющая изоморфные компоненты $\mathfrak{d}_{n-1}$-модуля $V_{m}$, содержит только факторы, последние две компоненты веса которых наследуются из модуля $T_{m}$, т. е. они имеют вид $m_{n-1}$ и $m_{n}$ (см. $[3$, теорема 4.3]). Когда мы говорим о представлениях $\mathcal{L}(\lambda)$ категории $\mathcal{L}$, обозначения этих параметров уже не имеют принципиального значения, поэтому можно обозначить их $\lambda_{n}$ и $\lambda_{n+1}$. В дальнейшем будем считать $m_{n-1}=\lambda_{n}$ и $m_{n}=\lambda_{n+1}$.

Проинтегрируем представление $\mathcal{L}(\lambda)$ алгебры Ли $\mathfrak{d}_{n-1 / 2}$ из категории $\mathcal{L}$ до представления ее группы Ли. Ввиду экспоненциальности ее группы Ли (см. предложение $3.1,(\mathrm{i})$ ) получаемое в результате интегрирования представление $D_{n-1 / 2}$ будет комплексно-аналитическим конечномерным представлением, для которого мы сохраним обозначение $\mathcal{L}(\lambda)$.

Поскольку мы рассматриваем регулярное вложение $D_{n-1}$ в $D_{n-1 / 2}$, задача описания спектра $\left.\mathcal{L}(\lambda)\right|_{D_{n-1}}$ эквивалентна описанию весов полустарших векторов, принадлежащих модулю $\mathcal{L}(\lambda)$. В ходе доказательства теоремы 2.1 для серии $D$ (см. [3, с. 176]) было показано, что фактор фильтрации $f$, порожденный образом квазистаршего веса $q_{\lambda}$, где $\lambda \rightarrow m$, порождается как $\mathfrak{d}_{n-1}$-модуль образами полустарших векторов $\omega(\mu, \lambda, m)$, где $\mu \rightarrow \lambda \rightarrow m$, т. е. выполняется

\footnotetext{
${ }^{3} \mathrm{~B}$ работе [3] применялось обозначение $q_{\lambda, m}$.
} 
система неравенств

$$
\begin{aligned}
\lambda_{1} \geqslant \mu_{1} \geqslant \lambda_{2} \geqslant \cdots \geqslant \lambda_{n-2} & \geqslant \mu_{n-2} \geqslant \max \left(\lambda_{n-1}, m_{n}\right) \\
& \geqslant \lambda_{n-1} \geqslant \mu_{n-1} \geqslant \lambda_{n-1}+m_{n}-\min \left(\mu_{n-2}, m_{n-1}\right) .
\end{aligned}
$$

Следовательно,

$$
\left.\mathcal{L}\left(\lambda_{1}, \ldots, \lambda_{n-1}, m_{n-1}, m_{n}\right)\right|_{D_{n-1}}=\bigoplus_{\mu \rightarrow \lambda \rightarrow m} T_{\mu}^{D_{n-1}},
$$

где прямая сумма берется по всем весам $\mu \in P_{n-1}^{D}$, подчиненным весам $\lambda \in P_{n-1}^{D}$ и $m \in P_{n}^{D}$. Формула (4.1) есть не что иное, как правило ветвления для редукции $D_{n-1 / 2} \downarrow D_{n-1}$.

В данном параграфе мы рассмотрим представления $\mathcal{L}(\lambda)$ групп Ли $D_{n-1 / 2}$ со старшими весами, последние две компоненты веса которых совпадают. Формулу для характеров $\mathcal{L}(\lambda)$ в случае произвольного веса нам получить не удалось. Это связано с тем, что правило ветвления (4.1) содержит такие параметры, $\operatorname{kak} \max \left(\lambda_{n-1}, \mu_{n}\right)$ и $\min \left(\mu_{n-2}, m_{n-1}\right)$, что приводит к рассмотрению большого количества случаев.

ПРеДЛОЖЕНИЕ 4.1. Характер $\operatorname{ch} \mathcal{L}(\lambda)$ представления $\mathcal{L}(\lambda)$ группы Ли $D_{n-1 / 2}$ однозначно определяется своим ограничением на $D_{n-1}$. Пусть $g \in D_{n-1 / 2}, g=g_{s} g_{u}-$ мультипликативное разложение Жордана, $g_{s} u g_{u}-$ соответственно полупростая и унипотентная матрицы. Тогда

$$
[\operatorname{ch} \mathcal{L}(\lambda)](g)=[\operatorname{ch} \mathcal{L}(\lambda)]\left(g_{s}\right)
$$

ДокАЗАтельство. Поскольку $D_{n-1 / 2}$ - замкнутая подгруппа группы $D_{n}$, компоненты $g_{s}, g_{u}$ принадлежат $D_{n-1 / 2}$. Так как компоненты Жордана коммутируют, можно (преобразованием подобия) привести одновременно $L(\lambda)\left(g_{s}\right)$ к диагональному, а $L(\lambda)\left(g_{u}\right)$ к верхнетреугольному виду с единицами на главной диагонали. В силу условия гомоморфизма имеем $L(\lambda)(g)=L(\lambda)\left(g_{s}\right) \cdot L(\lambda)\left(g_{u}\right)$, откуда вытекает, что собственные значения операторов $L(\lambda)(g)$ и $L(\lambda)\left(g_{s}\right)$ совпадают. Следовательно,

$$
[\operatorname{ch} \mathcal{L}(\lambda)](g)=\operatorname{tr} \mathcal{L}(\lambda)(g)=\operatorname{tr} \mathcal{L}(\lambda)\left(g_{s}\right)=[\operatorname{ch} \mathcal{L}(\lambda)]\left(g_{s}\right)
$$

Отметим, что $g_{s} \in D_{n-1}$ по предложению 3.1 .

Tеорема 4.1. Пусть $g \in D_{n-1 / 2},\left\{z_{1}, z_{2}, \ldots, z_{n-1}, 1,1, z_{n-1}^{-1}, \ldots, z_{2}^{-1}, z_{1}^{-1}\right\}-$ полный набор собственных значений $g$. Тогда характер представления $\mathcal{L}_{\left(\lambda_{1}, \lambda_{2}, \ldots, \lambda_{n-1}, \lambda_{n}, \lambda_{n}\right)}$ группь Ли $D_{n-1 / 2}$ со старшим весом $\left(\lambda_{1}, \lambda_{2}, \ldots, \lambda_{n-1}\right.$, $\left.\lambda_{n}, \lambda_{n}\right) \in \widetilde{P}_{n+1}$ может быть вычислен по формуле

$$
\left[\operatorname{ch} \mathcal{L}_{\left(\lambda_{1}, \lambda_{2}, \ldots, \lambda_{n-1}, \lambda_{n}, \lambda_{n}\right)}^{D_{n-1 / 2}}\right](g)=\frac{S_{1}(\lambda)+S_{2}(\lambda)}{S_{1}(0)},
$$


¿əe

$$
\begin{aligned}
S_{1} & =\left|\begin{array}{ccccc}
z_{1}^{\tau_{1}}-z_{1}^{-\tau_{1}} & \ldots & z_{1}^{\tau_{n-2}}-z_{1}^{-\tau_{n-2}} & \left(z_{1}^{\tau_{n-1}}+z_{1}^{-\tau_{n-1}}\right)\left(z_{1}^{1 / 2}-z_{1}^{-1 / 2}\right) & z_{1}^{\tau_{n}}-z_{1}^{-\tau_{n}} \\
\ldots & \ldots & \ldots & \ldots & \ldots \\
z_{n-1}^{\tau_{1}}-z_{n-1}^{-\tau_{1}} & \ldots & z_{n-1}^{\tau_{n-2}}-z_{n-1}^{-\tau_{n-2}} & \left(z_{n-1}^{\tau_{n-1}}+z_{n-1}^{-\tau_{n-1}}\right)\left(z_{n-1}^{1 / 2}-z_{n-1}^{-1 / 2}\right) & z_{n-1}^{\tau_{n}}-z_{n-1}^{-\tau_{n}} \\
1 & \ldots & 1 & 0 & 1
\end{array}\right|, \\
S_{2} & =\left|\begin{array}{ccccc}
z_{1}^{\tau_{1}}+z_{1}^{-\tau_{1}} & \ldots & z_{1}^{\tau_{n-2}}+z_{1}^{-\tau_{n-2}} & \left(z_{1}^{\tau_{n-1}}-z_{1}^{-\tau_{n-1}}\right)\left(z_{1}^{1 / 2}-z_{1}^{-1 / 2}\right) & z_{1}^{\tau_{n}}+z_{1}^{-\tau_{n}} \\
\ldots & \ldots & \ldots & \ldots & \ldots \\
z_{n-1}^{\tau_{1}}+z_{n-1}^{-\tau_{1}} & \ldots & z_{n-1}^{\tau_{n-2}}+z_{n-1}^{-\tau_{n-2}} & \left(z_{n-1}^{\tau_{n-1}}-z_{n-1}^{-\tau_{n-1}}\right)\left(z_{n-1}^{1 / 2}-z_{n-1}^{-1 / 2}\right) & z_{n-1}^{\tau_{n}}+z_{n-1}^{-\tau_{n}} \\
1 & \ldots & 1 & 0 & 1
\end{array}\right|
\end{aligned}
$$

и $\tau_{i}$ выражаются через $\lambda_{i}$ следующим образом:

$$
\begin{array}{cl}
\tau_{i}=\lambda_{i}+n-i-\frac{1}{2}, & i=1,2, \ldots, n-2, \\
\tau_{n-1}=\lambda_{n-1}, & \tau_{n}=\lambda_{n}+\frac{1}{2} .
\end{array}
$$

ДокАЗАТЕЛЬСтво. Согласно предложению 4.1 мы можем отождествлять элемент $g \in D_{n-1 / 2}$ с его компонентой Жордана $g_{s} \in D_{n-1}$. Исходя из правила ветвления для редукции $D_{n-1 / 2} \downarrow D_{n-1}$, имеем

$$
\left[\operatorname{ch} \mathcal{L}_{\left(\lambda_{1}, \lambda_{2}, \ldots, \lambda_{n}, \lambda_{n+1}\right)}^{D_{n-1 / 2}}\right](g)=\sum_{\mu \rightarrow \lambda}\left[\operatorname{ch} T_{\left(\mu_{1}, \mu_{2}, \ldots, \mu_{n-1}\right)}^{D_{n-1}}\right](g) .
$$

Напомним, что условие подчинения $\mu \rightarrow \lambda$ можно записать в виде следующей диаграммы, в которой стрелка соответствует знаку неравенства $\geqslant$ :

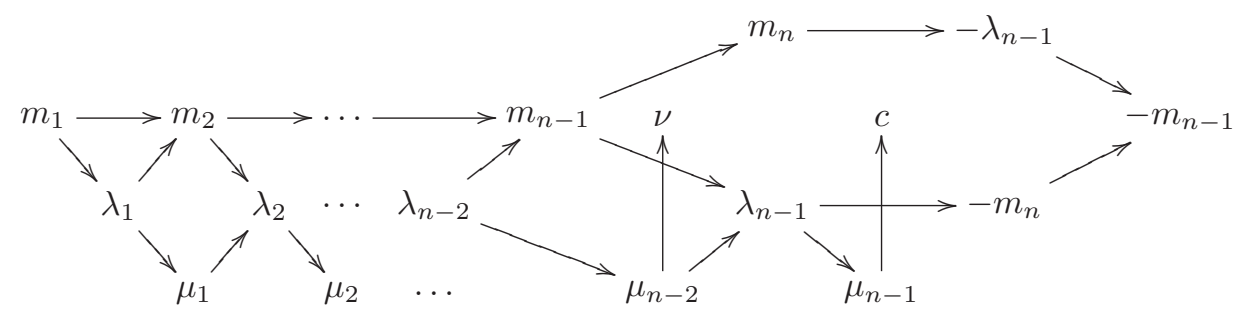

здесь ${ }^{4} \nu=\max \left(m_{n}, \lambda_{n-1}\right), c=\lambda_{n-1}+m_{n}-\min \left(m_{n-1}, \mu_{n-2}\right)$. Пусть теперь $\lambda_{n}=$ $\lambda_{n+1}$, т. е. в терминах диаграммы $m_{n-1}=m_{n}$. Имеем $\nu=\max \left(m_{n}, \lambda_{n-1}\right)=m_{n}$, так как $m_{n-1} \geqslant \lambda_{n-1}$. Тогда $m_{n} \leqslant \mu_{n-2} \leqslant \lambda_{n-2}$. Далее,

$$
\begin{aligned}
c & =\lambda_{n-1}+m_{n}-\min \left(m_{n-1}, \mu_{n-2}\right)=\lambda_{n-1}+m_{n}-\min \left(m_{n}, \mu_{n-2}\right) \\
& =\lambda_{n-1}+m_{n}-m_{n}=\lambda_{n-1} .
\end{aligned}
$$

Таким образом, $c=\lambda_{n-1} \leqslant \mu_{n-1} \leqslant \lambda_{n-1}$, и, значит, $\mu_{n-1}=\lambda_{n-1}$. Тогда формула (4.2) принимает следующий вид:

$$
\left[\operatorname{ch} \mathcal{L}_{\left(\lambda_{1}, \lambda_{2}, \ldots, \lambda_{n}, \lambda_{n}\right)}^{D_{n-1 / 2}}\right](g)=\sum_{\mu_{1}=\lambda_{2}}^{\lambda_{1}} \sum_{\mu_{2}=\lambda_{3}}^{\lambda_{2}} \ldots \sum_{\mu_{n-2}=\lambda_{n}}^{\lambda_{n-2}} \sum_{\mu_{n-1}=\lambda_{n-1}}^{\lambda_{n-1}}\left[\operatorname{ch} T_{\mu}^{D_{n-1}}\right](g) .
$$

${ }^{4}$ Для наглядности мы включили в диаграмму старший вес $m \in P_{n}$ такой, что $\mu \rightarrow \lambda \rightarrow m$. Последние две компоненты веса $m$ являются также компонентами веса $\lambda: m_{n-1}=\lambda_{n}$ и $m_{n}=\lambda_{n+1}$. 
Согласно формуле Вейля для характеров неприводимых представлений группы Ли $\mathrm{SO}(2 n-2)$ (см. [10, формулы $(32)-(34)]$; в обозначениях Вейля $z_{i}=e^{2 \pi i \varphi_{i}}$ и $l_{i}$ имеют то же значение, что и $q_{i}$ ниже) имеем

$$
\left[\operatorname{ch} T_{\left(\mu_{1}, \mu_{2}, \ldots, \mu_{n-1}\right)}^{D_{n-1}}\right](g)=\frac{\xi_{0}\left(q_{1}, \ldots, q_{n-1}\right)+\xi_{1}\left(q_{1}, \ldots, q_{n-1}\right)}{\xi_{0}(n-2, n-3, \ldots, 1,0)},
$$

где

$$
\begin{gathered}
\xi_{0}\left(q_{1}, \ldots, q_{n-1}\right)=\operatorname{det}\left(z_{i}^{q_{j}}+z_{i}^{-q_{j}}\right)_{i, j=1}^{n-1}, \\
\xi_{1}\left(q_{1}, \ldots, q_{n-1}\right)=\operatorname{det}\left(z_{i}^{q_{j}}-z_{i}^{-q_{j}}\right)_{i, j=1}^{n-1}, \\
q_{i}=\mu_{i}+n-1-i, \quad i=1,2, \ldots, n-1 .
\end{gathered}
$$

Для удобства обозначим $\xi_{0}(n-2, n-3, \ldots, 1,0)=\Delta$. Тогда

$$
\begin{aligned}
{\left[\operatorname{ch} T_{\mu}^{D_{n-1}}\right](g)=} & \frac{1}{\Delta} \sum_{\mu_{1}=\lambda_{2}}^{\lambda_{1}} \sum_{\mu_{2}=\lambda_{3}}^{\lambda_{2}} \ldots \sum_{\mu_{n-2}=\lambda_{n}=m_{n}}^{\lambda_{n-2}} \sum_{\mu_{n-1}=\lambda_{n-1}=\lambda_{n}}^{\lambda_{n-1}}\left(\xi_{0}+\xi_{1}\right) \\
= & \frac{1}{\Delta}\left[\sum_{\mu_{1}=\lambda_{2}}^{\lambda_{1}} \ldots \sum_{\mu_{n-2}=\lambda_{n-1}}^{\lambda_{n-2}} \sum_{\mu_{n-1}=\lambda_{n-1}}^{\lambda_{n-1}} \xi_{0}\left(q_{1}, \ldots, q_{n-1}\right)\right. \\
& \left.+\sum_{\mu_{1}=\lambda_{2}}^{\lambda_{1}} \ldots \sum_{\mu_{n-2}=\lambda_{n-1}}^{\lambda_{n-2}} \sum_{\mu_{n-1}=\lambda_{n-1}}^{\lambda_{n-1}} \xi_{1}\left(q_{1}, \ldots, q_{n-1}\right)\right]=\frac{1}{\Delta}\left(\widetilde{S_{0}}+\widetilde{S_{1}}\right),
\end{aligned}
$$

где $\widetilde{S_{0}}$ и $\widetilde{S_{1}}-(n-1)$-кратные суммы, которые мы вычислим отдельно. Сумма $\widetilde{S_{0}}$ дает определитель

$$
\left|\begin{array}{cccc}
C\left(z_{1}^{\lambda_{1}+n-2}\right)+\cdots+C\left(z_{1}^{\lambda_{2}+n-2}\right) & \ldots & C\left(z_{1}^{\lambda_{n-2}+1}\right)+\cdots+C\left(z_{1}^{\lambda_{n}+1}\right) & C\left(z_{1}^{\lambda_{n-1}}\right) \\
\cdots & \ldots & & \ldots \\
C\left(z_{n-1}^{\lambda_{1}+n-2}\right)+\cdots+C\left(z_{n-1}^{\lambda_{2}+n-2}\right) & \ldots & C\left(z_{n-1}^{\lambda_{n-2}+1}\right)+\cdots+C\left(z_{n-1}^{\lambda_{n}+1}\right) & C\left(z_{n-1}^{\lambda_{n-1}}\right)
\end{array}\right|,
$$

где мы применяем обозначения $C\left(z^{\lambda}\right)=z^{\lambda}+z^{-\lambda}, S\left(z^{\lambda}\right)=z^{\lambda}-z^{-\lambda}$. В угловых координатах $z=e^{2 \pi i \varphi}$ компактной вещественной формы группы $\operatorname{SO}(2 n-2)$ эти функции сводятся к косинусам и синусам: $C\left(z^{\lambda}\right)=2 \cos (2 \pi \lambda \varphi), S\left(z^{\lambda}\right)=$ $2 i \sin (2 \pi \lambda \varphi)$ (ср. [10, с. 144]).

Дополним этот определитель порядка $n-1$ до определителя порядка $n$, не изменяя его значения, следующим образом: добавим $n$-ю строку, состоящую из нулей, а в качестве $n$-го столбца вставим столбец

$$
\begin{aligned}
& \left(C\left(z_{1}^{\lambda_{n}}\right)+\cdots+C\left(z_{1}^{\lambda_{n-1}+1}\right)+C\left(z_{1}^{\lambda_{n-1}-1}\right)+\cdots+C\left(z_{1}\right)+2, \ldots\right. \\
& \left.\quad \ldots, C\left(z_{n-1}^{\lambda_{n}}\right)+\cdots+C\left(z_{n-1}^{\lambda_{n-1}+1}\right)+C\left(z_{n-1}^{\lambda_{n-1}-1}\right)+\cdots+C\left(z_{n-1}\right)+2,1\right)^{t} .
\end{aligned}
$$

Далее, в полученном определителе порядка $n$ сделаем следующие преобразования столбцов: к первому столбцу прибавим все последующие, ко второму все последующие и т. д. Другими словами, к $i$-му столбцу прибавим $(i+1)$-й, 
$(i+2)$-й, $\ldots, n$-й для $i=1,2, \ldots, n-2$. Кроме того, к $n$-му столбцу прибавим $(n-1)$-й, а $(n-1)$-й столбец оставим без изменения. Получим определитель

$$
\left|\begin{array}{ccccc}
C\left(z_{1}^{\lambda_{1}+n-2}\right)+\cdots+2 & \ldots & C\left(z_{1}^{\lambda_{n-2}+1}\right)+\cdots+2 & C\left(z_{1}^{\lambda_{n-1}}\right) & C\left(z_{1}^{\lambda_{n}}\right)+\cdots+2 \\
\cdots & \ldots & \ldots & \ldots & \ldots \\
C\left(z_{n-1}^{\lambda_{1}+n-2}\right)+\cdots+2 & \ldots & C\left(z_{n-1}^{\lambda_{n-2}+1}\right)+\cdots+2 & C\left(z_{n-1}^{\lambda_{n-1}}\right) & C\left(z_{n-1}^{\lambda_{n}}\right)+\cdots+2 \\
1 & \ldots & 1 & 0 & 1
\end{array}\right|
$$

где сумма вида $C\left(z^{\lambda}\right)+\cdots+2$ включает в себя все слагаемые вида $C\left(z^{k}\right)$ при $0 \leqslant k \leqslant \lambda$.

Пользуясь формулой для суммы членов геометрической прогрессии, получим

$$
\left|\begin{array}{ccccc}
\frac{z_{1}^{\lambda_{1}+n-1}-z_{1}^{-\left(\lambda_{1}+n-2\right)}}{z_{1}-1}+1 & \ldots & \frac{z_{1}^{\lambda_{n-2}+2}-z_{1}^{-\left(\lambda_{n-2}+1\right)}}{z_{1}-1}+1 & C\left(z_{1}^{\lambda_{n-1}}\right) & \frac{z_{1}^{\lambda_{n}+1}-z_{1}^{-\lambda_{n}}}{z_{1}-1}+1 \\
\ldots & \ldots & \ldots & \ldots & \ldots \\
\frac{z_{n-1}^{\lambda_{1}+n-1}-z_{n-1}^{-\left(\lambda_{1}+n-2\right)}}{z_{n-1}-1}+1 & \ldots & \frac{z_{n-1}^{\lambda_{n-2}+2}-z_{n-1}^{-\left(\lambda_{n-2}+1\right)}}{z_{n-1}-1}+1 & C\left(z_{n-1}^{\lambda_{n-1}}\right) & \frac{z_{n-1}^{\lambda_{n}+1}-z_{n-1}^{-\lambda_{n}}}{z_{n-1}-1}+1 \\
1 & \ldots & 1 & 0 & 1
\end{array}\right|
$$

Для всех $i=1,2, \ldots, n-1$ вынесем из $i$-й строки множитель $\left(z_{i}-1\right)^{-1}$, а затем прибавим к $i$-й строке последнюю, умноженную на $1-z_{i}$. Обозначим $\tau_{i}=$ $\lambda_{i}+n-i-1 / 2$, где $i=1,2, \ldots, n-2, \tau_{n-1}=\lambda_{n-1}, \tau_{n}=\lambda_{n}+1 / 2$. Получим определитель

$$
\begin{aligned}
& \frac{1}{\prod_{i=1}^{n-1}\left(z_{i}-1\right)} \\
& \times\left|\begin{array}{ccccc}
z_{1}^{\tau_{1}+\frac{1}{2}}-z_{1}^{-\tau_{1}+\frac{1}{2}} & \ldots & z_{1}^{\tau_{n-2}+\frac{1}{2}}-z_{1}^{-\tau_{n-2}+\frac{1}{2}} & C\left(z_{1}^{\tau_{n-1}}\right)\left(z_{1}-1\right) & z_{1}^{\tau_{n}+\frac{1}{2}}-z_{1}^{-\tau_{n}+\frac{1}{2}} \\
\ldots & \ldots & \ldots & \ldots & \ldots \\
z_{n-1}^{\tau_{1}+\frac{1}{2}}-z_{n-1}^{-\tau_{1}+\frac{1}{2}} & \ldots & z_{n-1}^{\tau_{n-2}+\frac{1}{2}}-z_{n-1}^{-\tau_{n-2}+\frac{1}{2}} & C\left(z_{n-1}^{\tau_{n-1}}\right)\left(z_{n-1}-1\right) & z_{n-1}^{\tau_{n}+\frac{1}{2}}-z_{n-1}^{-\tau_{n}+\frac{1}{2}} \\
1 & \ldots & 1 & 0 & 1
\end{array}\right| .
\end{aligned}
$$

Из каждой $i$-й строки, $i=1,2, \ldots, n-1$, вынесем множитель $z_{i}^{1 / 2}$. Получим определитель

$$
\frac{1}{\prod_{i=1}^{n-1}\left(z_{i}^{\frac{1}{2}}-z_{i}^{-\frac{1}{2}}\right)}\left|\begin{array}{ccccc}
S\left(z_{1}^{\tau_{1}}\right) & \ldots & S\left(z_{1}^{\tau_{n-2}}\right) & C\left(z_{1}^{\tau_{n-1}}\right)\left(z_{1}^{\frac{1}{2}}-z_{1}^{-\frac{1}{2}}\right) & S\left(z_{1}^{\tau_{n}}\right) \\
\ldots & \ldots & \ldots & \ldots & \ldots \\
S\left(z_{n-1}^{\tau_{1}}\right) & \ldots & S\left(z_{n-1}^{\tau_{n-2}}\right) & C\left(z_{n-1}^{\tau_{n-1}}\right)\left(z_{n-1}^{\frac{1}{2}}-z_{n-1}^{-\frac{1}{2}}\right) & S\left(z_{n-1}^{\tau_{n}}\right) \\
1 & \ldots & 1 & 0 & 1
\end{array}\right|
$$

Сделав аналогичные преобразования для суммы $\widetilde{S_{1}}$, получим определитель

$$
\frac{1}{\prod_{i=1}^{n-1}\left(z_{i}^{\frac{1}{2}}-z_{i}^{-\frac{1}{2}}\right)}\left|\begin{array}{ccccc}
C\left(z_{1}^{\tau_{1}}\right) & \ldots & C\left(z_{1}^{\tau_{n-2}}\right) & S\left(z_{1}^{\tau_{n-1}}\right)\left(z_{1}^{\frac{1}{2}}-z_{1}^{-\frac{1}{2}}\right) & C\left(z_{1}^{\tau_{n}}\right) \\
\ldots & \ldots & \ldots & \ldots & \ldots \\
C\left(z_{n-1}^{\tau_{1}}\right) & \ldots & C\left(z_{n-1}^{\tau_{n-2}}\right) & S\left(z_{n-1}^{\tau_{n-1}}\right)\left(z_{n-1}^{\frac{1}{2}}-z_{n-1}^{-\frac{1}{2}}\right) & C\left(z_{n-1}^{\tau_{n}}\right) \\
1 & \ldots & 1 & 0 & 1
\end{array}\right| .
$$

Мы получили

$$
\left[\operatorname{ch} \mathcal{L}_{\lambda}^{D_{n-1 / 2}}\right](g)=\frac{S_{1}(\lambda)+S_{2}(\lambda)}{\prod_{i=1}^{n-1}\left(z_{i}^{\frac{1}{2}}-z_{i}^{-\frac{1}{2}}\right) \Delta} .
$$


Преобразуем знаменатель $\prod_{i=1}^{n-1}\left(z_{i}^{\frac{1}{2}}-z_{i}^{-\frac{1}{2}}\right) \Delta$ :

$$
\begin{aligned}
& \prod_{i=1}^{n-1}\left(z_{i}^{\frac{1}{2}}-z_{i}^{-\frac{1}{2}}\right) \Delta \\
& =2 \prod_{i=1}^{n-1}\left(z_{i}^{\frac{1}{2}}-z_{i}^{-\frac{1}{2}}\right)\left|\begin{array}{cccc}
z_{1}^{n-2}-z_{1}^{-(n-2)} & \ldots & z_{1}-z_{1}^{-1} & 1 \\
\ldots & \ldots & \ldots & \ldots \\
z_{n-1}^{n-2}-z_{n-1}^{-(n-2)} & \ldots & z_{n-1}-z_{n-1}^{-1} & 1
\end{array}\right| \\
& =2\left|\begin{array}{ccccc}
S\left(z_{1}^{n-\frac{3}{2}}\right)-S\left(z_{1}^{n-\frac{5}{2}}\right) & S\left(z_{1}^{n-\frac{5}{2}}\right)-S\left(z_{1}^{n-\frac{7}{2}}\right) & \ldots & S\left(z_{1}^{\frac{3}{2}}\right)-S\left(z_{1}^{\frac{1}{2}}\right) & S\left(z_{1}^{\frac{1}{2}}\right) \\
\ldots & \ldots & \ldots & \ldots \\
S\left(z_{n-1}^{n-\frac{3}{2}}\right)-S\left(z_{n-1}^{n-\frac{5}{2}}\right) & S\left(z_{n-1}^{n-\frac{5}{2}}\right)-S\left(z_{n-1}^{n-\frac{7}{2}}\right) & \ldots & S\left(z_{n-1}^{\frac{3}{2}}\right)-S\left(z_{n-1}^{\frac{1}{2}}\right) & S\left(z_{n-1}^{\frac{1}{2}}\right)
\end{array}\right|
\end{aligned}
$$

Дополним определитель порядка $n-1$ до определителя порядка $n$ следующим образом: снизу припишем строку нулей, а в последний столбец вставим $\left(z_{1}^{\frac{1}{2}}-z_{1}^{-\frac{1}{2}}, \ldots, z_{n-1}^{\frac{1}{2}}-z_{n-1}^{-\frac{1}{2}}, 1\right)^{t}$. Далее сделаем следующие преобразования столбцов: к $i$-му столбцу для $i=1,2, \ldots, n-3$ прибавим $(i+1)$-й, $(i+2)$-й, $\ldots$ $\ldots,(n-2)$-й, $n$-й (кроме $(n-1)$-го). Кроме того, к $n$-му столбцу прибавим $(n-1)$-й, а $(n-2)$-й столбец оставим без изменения. Множитель 2 внесем в $(n-1)$-й столбец. Получим определитель $S_{1}(0)$, равный

$$
\left|\begin{array}{ccccc}
z_{1}^{n-\frac{3}{2}}-z_{1}^{-\left(n-\frac{3}{2}\right)} & z_{1}^{n-\frac{5}{2}}-z_{1}^{-\left(n-\frac{5}{2}\right)} & \ldots & 2\left(z_{1}^{\frac{1}{2}}-z_{1}^{-\frac{1}{2}}\right) & z_{1}^{\frac{1}{2}}-z_{1}^{-\frac{1}{2}} \\
\ldots & \ldots & \ldots & \ldots & \ldots \\
z_{n-1}^{n-\frac{3}{2}}-z_{n-1}^{-\left(n-\frac{3}{2}\right)} & z_{n-1}^{n-\frac{5}{2}}-z_{n-1}^{-\left(n-\frac{5}{2}\right)} & \ldots & 2\left(z_{n-1}^{\frac{1}{2}}-z_{n-1}^{-\frac{1}{2}}\right) & z_{n-1}^{\frac{1}{2}}-z_{n-1}^{-\frac{1}{2}} \\
1 & 1 & \ldots & 0 & 1
\end{array}\right|
$$

Теорема доказана.

\section{§ 5. Размерности представлений $\mathcal{L}(\lambda)$ со старшими весами $\left(\lambda_{1}, \ldots, \lambda_{n-1}, \lambda_{n}, \lambda_{n}\right)$}

Размерность представления $\mathcal{L}(\lambda)$ можно вычислить по крайней мере двумя способами. Первый - предельным переходом в формуле для характера $\mathcal{L}(\lambda)$ при $g \rightarrow e$, второй - при использовании разложения

$$
\left.\mathcal{L}(\lambda)\right|_{D_{n-1}}=\bigoplus_{\mu \rightarrow \lambda} T_{\mu}
$$

и формулы Г. Вейля для размерностей неприводимых $\operatorname{SO}(2 n-2)$-модулей $T_{\mu}$, $\mu \in P_{n-1}$.

Tеорема 5.1. Пусть $\lambda \in \widetilde{P}_{n+1}, \lambda=\left(\lambda_{1}, \ldots, \lambda_{n-1}, \lambda_{n}, \lambda_{n}\right)$. Размерность представления $\mathcal{L}(\lambda)$ группь Ли $D_{n-1 / 2}$ вычисляется по бормуле

$$
\operatorname{dim} \mathcal{L}(\lambda)=\frac{d\left(\lambda_{1}, \lambda_{2}, \ldots, \lambda_{n-1}, \lambda_{n}\right)}{d(0,0, \ldots, 0,0)},
$$


¿əe

$$
d(\lambda)=\left|\begin{array}{cccccc}
\tau_{1}^{2 n-3} & \tau_{2}^{2 n-3} & \ldots & \tau_{n-2}^{2 n-3} & \left(\tau_{n-1}+\frac{1}{2}\right)^{2 n-3}-\left(\tau_{n-1}-\frac{1}{2}\right)^{2 n-3} & \tau_{n}^{2 n-3} \\
\tau_{1}^{2 n-5} & \tau_{2}^{2 n-5} & \ldots & \tau_{n-2}^{2 n-5} & \left(\tau_{n-1}+\frac{1}{2}\right)^{2 n-3}-\left(\tau_{n-1}-\frac{1}{2}\right)^{2 n-5} & \tau_{n}^{2 n-5} \\
\ldots & \ldots & \ldots & \ldots & \ldots & \ldots \\
\tau_{1} & \tau_{2} & \ldots & \tau_{n-2} & \left(\tau_{n-1}+\frac{1}{2}\right)-\left(\tau_{n-1}-\frac{1}{2}\right) & \tau_{n} \\
1 & 1 & \ldots & 1 & 0 & 1
\end{array}\right|,
$$

a $\tau_{i}$ определены в теореме 4.1.

ДоказАтельство. Размерность неприводимого представления $T_{\mu}$ со старшим весом $\mu \in P_{n-1}$ группы $\mathrm{SO}(2 n-2)$ выражается формулой Г. Вейля (см. [10, формула $\left.\left.\left(35^{\prime}\right)\right]\right)$

$$
\operatorname{dim} T_{\mu}^{D_{n-1}}=\frac{\xi\left(t_{1}, t_{2}, \ldots, t_{n-2}, t_{n-1}\right)}{\xi(n-2, n-3, \ldots, 1,0)},
$$

где

$$
\xi\left(t_{1}, t_{2}, \ldots, t_{n-2}, t_{n-1}\right)=\left|\begin{array}{cccc}
t_{1}^{2(n-2)} & t_{2}^{2(n-2)} & \ldots & t_{n-1}^{2(n-2)} \\
\ldots & \ldots & \ldots & \ldots \\
t_{1}^{2} & t_{2}^{2} & \ldots & t_{n-1}^{2} \\
1 & 1 & \ldots & 1
\end{array}\right|
$$

и $t_{k}=\mu_{k}+n-k-1, k=1,2, \ldots, n-1$. Применяя правило ветвления для редукции $D_{n-1 / 2} \downarrow D_{n-1}$ в случае, когда две последние компоненты веса $\lambda$ совпадают, и разложение (5.1), имеем

$$
\begin{array}{r}
\operatorname{dim} \mathcal{L}(\lambda)=\frac{1}{\Delta} \sum_{\mu_{1}=\lambda_{2}}^{\lambda_{1}} \sum_{\mu_{2}=\lambda_{3}}^{\lambda_{2}} \ldots \sum_{\mu_{n-2}=\lambda_{n}}^{\lambda_{n-2}} \sum_{\mu_{n-1}=\lambda_{n-1}}^{\lambda_{n-1}}\left|\begin{array}{cccc}
t_{1}^{2(n-2)} & t_{2}^{2(n-2)} & \ldots & t_{n-1}^{2(n-2)} \\
\ldots & \ldots & \ldots & \ldots \\
t_{1}^{2} & t_{2}^{2} & \ldots & t_{n-1}^{2} \\
1 & 1 & \ldots & 1
\end{array}\right| \\
=\frac{1}{\Delta}\left|\begin{array}{ccccc}
\sum_{t=\frac{1}{2}}^{\tau_{1}-\frac{1}{2}} t^{2(n-2)} & \sum_{t=\tau_{3}+\frac{1}{2}}^{\tau_{2}-\frac{1}{2}} t^{2(n-2)} & \ldots & \sum_{t=\tau_{n}+\frac{1}{2}}^{\tau_{n-2}} t^{2(n-2)} & \tau_{n-1}^{2(n-2)} \\
\sum_{t=\frac{1}{2}}^{\tau_{1}-\frac{1}{2}} t_{2}^{2(n-3)} & \sum_{t=\frac{1}{2}}^{\tau_{2}-\frac{1}{2}} t_{3} t^{2(n-3)} & \ldots & \sum_{t=\tau_{n}+\frac{1}{2}}^{\tau_{n-2}-\frac{1}{2}} t^{2(n-3)} & \tau_{n-1}^{2(n-3)} \\
\ldots & \ldots & \ldots & \ldots & \ldots \\
\sum_{t=\tau_{2}+\frac{1}{2}}^{\tau_{1}-\frac{1}{2}} t^{2} & \sum_{t=\tau_{3}+\frac{1}{2}}^{\tau_{2}-\frac{1}{2}} t^{2} & \ldots & \sum_{t=\tau_{n}+\frac{1}{2}}^{\tau_{n-2}-\frac{1}{2}} t^{2} & \tau_{n-1}^{2} \\
\tau_{1}-\tau_{2} & \tau_{2}-\tau_{3} & \ldots & \tau_{n-2}-\tau_{n} & 1
\end{array}\right| .
\end{array}
$$

Дополним определитель порядка $n-1$ до определителя порядка $n$ следующим образом: снизу припишем нулевую строку, а справа в качестве $n$-го столбца на последнее место вставим столбец

$$
\begin{gathered}
\left(\left(\tau_{n}-\frac{1}{2}\right)^{2(n-2)}+\cdots+\left(\tau_{n-1}+1\right)^{2(n-2)}+\left(\tau_{n-1}-1\right)^{2(n-2)}+\cdots+1, \ldots\right. \\
\left.\ldots,\left(\tau_{n}-\frac{1}{2}\right)^{2}+\cdots+\left(\tau_{n-1}+1\right)^{2}+\left(\tau_{n-1}-1\right)^{2}+\cdots+1, \tau_{n}-\frac{1}{2}, 1\right)^{t} .
\end{gathered}
$$

Значение определителя не изменится. В полученном определителе порядка $n$ прибавим к каждому столбцу, кроме последних двух, все последующие, 
т. е. к $i$-му столбцу для $i=1,2, \ldots, n-2$ прибавим $(i+1)$-й, $(i+2)$-й, $\ldots, n$-й. Кроме того, к $n$-му столбцу прибавим $(n-1)$-й, а $(n-1)$-й столбец оставим без изменения. Получим определитель

$$
\left|\begin{array}{ccccc}
S_{2(n-2)}\left(\lambda_{1}+n-2\right) & S_{2(n-2)}\left(\lambda_{2}+n-3\right) & \ldots & \lambda_{n-1}^{2(n-2)} & S_{2(n-2)}\left(\lambda_{n}\right) \\
\ldots & \ldots & \ldots & \ldots & \ldots \\
S_{2}\left(\lambda_{1}+n-2\right) & S_{2}\left(\lambda_{2}+n-3\right) & \ldots & \lambda_{n-1}^{2} & S_{2}\left(\lambda_{n}\right) \\
\lambda_{1}+n-1 & \lambda_{2}+n-2 & \ldots & 1 & \lambda_{n}+1 \\
1 & 1 & \ldots & 0 & 1
\end{array}\right|
$$

где введено обозначение $S_{k}(m)=1^{k}+2^{k}+\cdots+m^{k}$.

Вычтем из $(n-1)$-й строки последнюю и сделаем замену $z_{i}=\lambda_{i}+n-i-1$, $i=1,2, \ldots, n-1 ; z_{n}=\lambda_{n}$. Получим определитель

$$
\left|\begin{array}{ccccc}
S_{2(n-2)}\left(z_{1}\right) & S_{2(n-2)}\left(z_{2}\right) & \ldots & z_{n-1}^{2(n-2)} & S_{2(n-2)}\left(z_{n}\right) \\
\ldots & \ldots & \ldots & \ldots & \ldots \\
S_{2}\left(z_{1}\right) & S_{2}\left(z_{2}\right) & \ldots & z_{n-1}^{2} & S_{2}\left(z_{n}\right) \\
z_{1} & z_{2} & \ldots & 1 & z_{n} \\
1 & 1 & \ldots & 0 & 1
\end{array}\right| .
$$

Для вычисления последнего определителя докажем

ПреДложениЕ 5.1. Для определителей, элементы которых-четные степенные суммы $S_{2(k-2)}\left(\lambda_{i}\right)$, имеет место следующее тождество:

$$
\begin{aligned}
& \left|\begin{array}{cccc}
S_{2(n-2)}\left(\lambda_{1}\right) & S_{2(n-2)}\left(\lambda_{2}\right) & \ldots & S_{2(n-2)}\left(\lambda_{n}\right) \\
S_{2(n-3)}\left(\lambda_{1}\right) & S_{2(n-3)}\left(\lambda_{2}\right) & \ldots & S_{2(n-3)}\left(\lambda_{n}\right) \\
\ldots & \ldots & \ldots & \ldots \\
S_{2}\left(\lambda_{1}\right) & S_{2}\left(\lambda_{2}\right) & \ldots & S_{2}\left(\lambda_{n}\right) \\
\lambda_{1} & \lambda_{2} & \ldots & \lambda_{n} \\
1 & 1 & \ldots & 1
\end{array}\right| \\
& =\frac{1}{(2 n-3) ! !}\left|\begin{array}{cccc}
\left(\lambda_{1}+\frac{1}{2}\right)^{2 n-3} & \left(\lambda_{2}+\frac{1}{2}\right)^{2 n-3} & \ldots & \left(\lambda_{n}+\frac{1}{2}\right)^{2 n-3} \\
\left(\lambda_{1}+\frac{1}{2}\right)^{2 n-5} & \left(\lambda_{2}+\frac{1}{2}\right)^{2 n-5} & \ldots & \left(\lambda_{n}+\frac{1}{2}\right)^{2 n-5} \\
\ldots & \ldots & \ldots & \ldots \\
\lambda_{1}+\frac{1}{2} & \lambda_{2}+\frac{1}{2} & \ldots & \lambda_{n}+\frac{1}{2} \\
1 & 1 & \ldots & 1
\end{array}\right| .
\end{aligned}
$$

ДоказАтельство. Доказываемое тождество сразу вытекает из сравнения

$$
S_{2(n-2)}(\lambda) \equiv \frac{(\lambda+1 / 2)^{2 n-3}}{(2 n-3)}\left(\bmod L\left(\left(\lambda+\frac{1}{2}\right),\left(\lambda+\frac{1}{2}\right)^{3}, \ldots,\left(\lambda+\frac{1}{2}\right)^{2 n-5}\right)\right) .
$$

Покажем, что $S_{2(n-2)}(\lambda)$ является многочленом нечетной степени от $z=$ $\lambda+\frac{1}{2}$ со старшим членом $\frac{1}{(2 n-3)} z^{2 n-3}$. Воспользуемся известным соотношением, связывающим степенные суммы с числами Бернулли [12, теорема 2]:

$$
(k+1) S_{k}(\lambda)=(\lambda+1+B)^{k+1}-B^{k+1}=\left(z+\frac{1}{2}+B\right)^{k+1}-B^{k+1}, \quad k \in 2 \mathbb{Z} .
$$


Отсюда, учитывая, что $B_{1}=-\frac{1}{2}$, а остальные числа Бернулли с нечетными индексами равны нулю, получим

$$
\begin{aligned}
(k+1) S_{k}(\lambda)= & \left(z+\frac{1}{2}\right)^{k+1}-\frac{k+1}{2}\left(z+\frac{1}{2}\right)^{k} \\
& +C_{k+1}^{2} B_{2}\left(z+\frac{1}{2}\right)^{k-1}+\cdots+C_{k+1}^{k} B_{k}\left(z+\frac{1}{2}\right) .
\end{aligned}
$$

Далее, из рекуррентного соотношения для чисел Бернулли $(1+B)^{m}=B^{m}$, $m \geqslant 2[12$, гл. $\mathrm{V}, \S 8$, теорема 1$]$, вытекает $(x+B)^{m}=(x-1-B)^{m}, m \geqslant 1$, откуда

$$
\begin{aligned}
& (k+1) S_{k}(\lambda)=(\lambda-B)^{k+1}+B^{k+1}=\left(z-\frac{1}{2}-B\right)^{k+1}+B^{k+1} \\
& =\left(z-\frac{1}{2}\right)^{k+1}+\frac{k+1}{2}\left(z-\frac{1}{2}\right)^{k}+C_{k+1}^{2} B_{2}\left(z-\frac{1}{2}\right)^{k-1}+\cdots+C_{k+1}^{k} B_{k}\left(z-\frac{1}{2}\right) .
\end{aligned}
$$

Осталось заметить, что так как $k$ - четное число, то после суммирования равенств (5.3) и (5.4) все четные степени $z$ аннулируются. Предложение доказано.

Возвращаясь к доказательству теоремы 5.1, представим элементы предпоследнего столбца определителя (5.2) как разности степенных сумм

$$
z_{n-1}^{2 i}=S_{2 i}\left(z_{n-1}\right)-S_{2 i}\left(z_{n-1}-1\right)
$$

и, применяя предложение 5.1, получим следующее представление числителя в формуле для размерностей:

$$
\overline{(2 n-3) ! !}\left|\begin{array}{ccccc}
\tau_{1}^{2 n-3} & \tau_{2}^{2 n-3} & \ldots & \left(\tau_{n-1}+\frac{1}{2}\right)^{2 n-3}-\left(\tau_{n-1}-\frac{1}{2}\right)^{2 n-3} & \tau_{n}^{2 n-3} \\
\tau_{1}^{2 n-5} & \tau_{2}^{2 n-5} & \ldots & \left(\tau_{n-1}+\frac{1}{2}\right)^{2 n-5}-\left(\tau_{n-1}-\frac{1}{2}\right)^{2 n-5} & \tau_{n}^{2 n-5} \\
\ldots & \ldots & \ldots & \ldots & \ldots \\
\tau_{1} & \tau_{2} & \ldots & \left(\tau_{n-1}+\frac{1}{2}\right)-\left(\tau_{n-1}-\frac{1}{2}\right) & \tau_{n} \\
1 & 1 & \ldots & 0 & 1
\end{array}\right|
$$

Для завершения доказательства осталось воспользоваться легко проверяемым тождеством определителей

$$
\begin{aligned}
& 2^{n-2}\left|\begin{array}{cccc}
(n-2)^{2(n-2)} & (n-3)^{2(n-2)} & \ldots & 1^{2(n-2)} \\
\ldots & \ldots & \ldots & \ldots \\
(n-2)^{4} & (n-3)^{4} & \ldots & 1^{4} \\
(n-2)^{2} & (n-3)^{2} & \ldots & 1^{2}
\end{array}\right| \\
& =\left|\begin{array}{cccc}
\left(n-\frac{3}{2}\right)^{2(n-2)} & \ldots & \left(\frac{3}{2}\right)^{2(n-2)} & \left(\frac{1}{2}\right)^{2(n-2)} \\
\ldots & \ldots & \ldots & \ldots \\
\left(n-\frac{3}{2}\right)^{2} & \ldots & \left(\frac{3}{2}\right)^{2} & \left(\frac{1}{2}\right)^{2} \\
1 & \ldots & 1 & 1
\end{array}\right| .
\end{aligned}
$$

Теорема доказана. 
ЗАмечАниЕ 5.1. Из правила ветвления для редукции $D_{n-1 / 2} \downarrow D_{n-1}$ очевидно, что два представления группы $D_{n-1 / 2}$ со старшими весами $\lambda$ и $\lambda^{\prime}$, $\lambda, \lambda^{\prime} \in \widetilde{P}_{n+1}$, изоморфны тогда и только тогда, когда $\lambda=\lambda^{\prime}$ (действительно, при $\lambda \neq \lambda^{\prime}$ модули $\mathcal{L}(\lambda)$ и $\mathcal{L}\left(\lambda^{\prime}\right)$ не изоморфны уже как $D_{n-1}$-модули). Таким образом, представления категории $\mathcal{L}$ с точностью до изоморфизма описываются своими старшими весами, а значит, и характерами.

То, что сумма $\sum_{\mu \rightarrow \lambda}\left[\operatorname{ch} T_{\left(\mu_{1}, \mu_{2}, \ldots, \mu_{n-1}\right)}^{D_{n-1}}\right](g)$ в правой части равенства $(4.2)$ сворачивается с точностью до постоянного множителя в сумму двух определителей $S_{1}(\lambda)$ и $S_{2}(\lambda)$, является еще одним нетривиальным проявлением "скрытой симметрии" в смысле И. М. Гельфанда и А. В. Зелевинского [11].

Наконец, мы получили еще одно подтверждение того, что группа Ли $D_{n-1 / 2}$ должна быть отнесена именно к четной ортогональной серии (а не к серии $B$, как можно было бы предположить из сравнения цепочек редукций, рассмотренных в конце §2). Действительно, доказанная нами в теореме 4.1 формула для характеров представлений категории $\mathcal{L}$ группы Ли $D_{n-1 / 2}$ имеет вейлевский вид, если ее сравнивать с формулой для характеров неприводимых представлений простой группы Ли $D_{n}$. Напомним, что из всех классических простых групп Ли только в серии $D$ в числителе формулы для характеров имеется сумма двух определителей, зависящих от компонент старшего веса.

\section{Список литературы}

1. В.В. Штепин, "Промежуточные алгебры Ли и их конечномерные представления”, Изв. РАН. Сер. матем., 57:6 (1993), 176-198; англ. пер.: V. V. Shtepin, "Intermediate Lie algebras and their finite-dimensional representations", Russian Acad. Sci. Izv. Math., 43:3 (1994), 559-579.

2. В.В. Штепин, "Промежуточная ортогональная алгебра Ли $\mathfrak{b}_{n-1 / 2}$ и ее конечномерные представления", Изв. РАН. Сер. матем., 62:3 (1998), 201-223; англ. пер.: V. V. Shtepin, "The intermediate orthogonal Lie algebra $\mathfrak{b}_{n-1 / 2}$ and its finite-dimensional representations", Izv. Math., 62:3 (1998), 627-648.

3. В.В. Штепин, "Промежуточная алгебра Ли $\mathfrak{d}_{n-1 / 2}$, весовая схема и конечномерные представления со старшим весом", Изв. РАН. Сер. матем., 68:2 (2004), 159-190; англ. пер.: V. V. Shtepin, "The intermediate Lie algebra $\mathfrak{d}_{n-1 / 2}$, the weight scheme and finite-dimensional representations with highest weight", Izv. Math., 68:2 (2004), 375-404.

4. Г. Вейль, Теория групп и квантовая механика, Наука, М., 1986; пер. с англ.: H. Weyl, Gruppentheorie und Quantenmechanik, S. Hirzel, Leipzig, 1931.

5. И. М. Гельфанд, М. Л. Цетлин, "Конечномерные представления группы ортогональных матриц", Докл. АН СССР, 71:6 (1950), 1017-1020.

6. Д. П. Желобенко, "Классические группы. Спектральный анализ конечномерных представлений", УМH, 17:1 (1962), 27-120; англ. пер.: D. P. Zhelobenko, "The classical groups. Spectral analysis of their finite-dimensional representations", Russian Math. Surveys, 17:1 (1962), 1-94.

7. А. Барут, Р. Рончка, Теория представлений групп и ее приложения, т. 1, Мир, M., 1980; пер. с англ.: А. O. Barut, R. Raczka, Theory of group representations and applications, Polish Scientific Publ., Warsaw, 1977.

8. A. M. Bincer, "Bases in $\mathrm{Sp}(2 n)$ representation space" (Cocoyoc, 1980), Lecture Notes in Phys., 135 (1980), 459-463. 
9. В. В. Штепин, "Разделение кратных точек спектра в редукции $\operatorname{sp}(2 n) \downarrow \operatorname{sp}(2 n-2)$ ", Функи. анализ и его прил., 20:4 (1986), 93-95; англ. пер.: V. V. Shtepin, "Separation of multiple points of spectrum in the reduction $\operatorname{sp}(2 n) \downarrow \operatorname{sp}(2 n-2) "$, Funct. Anal. Appl., 20:4 (1986), 336-338.

10. Г. Вейль, “Теория представлений непрерывных полупростых групп при помощи линейных преобразований”, Избранные труды: Математика. Теоретическая физика, Наука, М., 1984, 100-197.

11. И. М. Гельфанд, А. В. Зелевинский, "Модели представлений классических групп и их скрытые симметрии", Функи. анализ и его прил., 18:3 (1984), 14-31; англ. пер.: I. M. Gel'fand, A. V. Zelevinskii, "Models of representations of classical groups and their hidden symmetries", Funct. Anal. Appl., 18:3 (1984), 183-198.

12. З. И. Боревич, И. Р. Шафаревич, Теория чисел, 3-е изд., Наука, М., 1985; англ. пер. 1-го изд.: А. I. Borevich, I. R. Shafarevich, Number theory, Academic Press, New York-London, 1966.

ВАДИМ ВЛАДИМИРОВИЧ ШТЕПИН

Поступило в редакцию

(VAdim V. Shtepin)

11.07 .2012

Донецкий национальный университет

E-mail: vadim.shtepin@gmail.com

ДАниИл ЛЕОНИДОВИч КоНАшЕНКОВ

(Danill L. Konashenkov)

Донецкий национальный университет

E-mail: konashenkov@rambler.ru 